\title{
Airfoil Performance at Low Reynolds Numbers in the Presence of Periodic Disturbances
}

\section{S. Yarusevych}

University of Toronto

\section{J. G. Kawall}

Ryerson University

\section{P. E. Sullivan}

University of Toronto

Yarusevych, S., Kawall, J. G., \& Sullivan, P. E. (2005). Airfoil performance at low Reynolds numbers in the presence of periodic disturbances. ASME: Journal of Fluids Engineering, 128(3), 587-595.

doi:10.1115/1.2175165

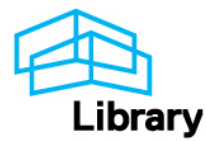




\section{Airfoil Performance at Low Reynolds Numbers in the Presence of Periodic Disturbances}

\author{
J. G. Kawall \\ Ryerson University, \\ Department of Mechanical and Industrial \\ Engineering, \\ 350 Victoria St., \\ Toronto, Ontario, M5B 2K3, Canada \\ e-mail: gkawall@ryerson.ca \\ P. E. Sullivan \\ University of Toronto, \\ Department of Mechanical and Industrial \\ Engineering, \\ 5 King's College Rd., \\ Toronto, Ontario, M5S 3G8, Canada \\ e-mail: sullivan@mie.utoronto.ca
}

The boundary-layer separation and wake structure of a NACA 0025 airfoil and the effect of external excitations in presence of structural vibrations on airfoil performance were studied experimentally. Wind tunnel experiments were carried out for three Reynolds numbers and three angles of attack, involving hot-wire measurements and complementary surface flow visualization. The results establish that external acoustic excitation at a particular frequency and appropriate amplitude suppresses or reduces the separation region and decreases the airfoil wake, i.e., produces an increase of the lift and/or decrease of the drag. The acoustic excitation also alters characteristics of the vortical structures in the wake, decreasing the vortex length scale and coherency. Optimum excitation frequencies were found to correlate with the fundamental frequencies of the naturally amplified disturbances in the separated shear layer. The results suggest that acoustic waves play a dominant role in exciting the separated shear layer of the airfoil. Moreover, low-frequency structural vibrations are found to have a significant effect on airfoil performance, as they enhance the sound pressure levels within the test section. [DOI: 10.1115/1.2175165]

Keywords: boundary-layer separation, low-Reynolds-number flow, wake formation, vortex shedding, flow control, acoustic excitation

\section{Introduction}

The performance of airfoils at low Reynolds numbers has been of interest in connection with a wide range of applications, including the operation of aircraft at low speeds and the design of micro air vehicles, compressor blades, wind turbines, and inboard sections of helicopter rotors. Several investigators have studied airfoil performance in the low Reynolds number region (e.g., $[1,2])$. Their findings indicate that serious aerodynamic problems occur below Reynolds number of about 200,000. Specifically, the laminar boundary layer on the upper surface of the airfoil is subjected to an adverse pressure gradient, even at low angles of attack. This often results in laminar boundary-layer separation and formation of a shear layer. At lower Reynolds numbers, the separated shear layer does not reattach to the airfoil surface, and a large wake is formed. In contrast, at higher Reynolds numbers, the separated shear layer may reattach to the airfoil surface, resulting in the formation of a separation bubble. It should be stressed that, in both cases, laminar separation has a significant detrimental effect on airfoil lift and drag. Therefore, it is of interest to improve airfoil performance by introducing a flow control mechanism to energize the boundary layer sufficiently to overcome the adverse pressure gradient and reduce or suppress the separation region. One method of flow control excites the boundary layer with an acoustic source. A number of studies have demonstrated that acoustic excitation applied at an appropriate frequency and amplitude reduces the separation region and improves airfoil characteristics [3-11]. However, the complex physical mechanism responsible for this is not yet known in detail.

At angles of attack below and in the vicinity of the stall angle, some experimental results [4-7] suggest that the optimum effect

${ }^{1}$ Corresponding author.

Contributed by the Fluids Engineering Division of ASME for publication in the Journal of Fluids EngineERING. Manuscript received March 4, 2005; final manuscript received October 10, 2005. Review conducted by Joseph Katz. occurs when the excitation frequency matches the instability frequency of the separated shear layer. However, at angles of attack well above the stall angle, Hsiao et al. [7] have found that the most effective excitation frequency matches the vortex shedding frequency in the airfoil wake. Thus, the effect of the excitation and the corresponding control mechanism appear to be different for different ranges of the angle of attack. Furthermore, it has been concluded [5-9] that the effect and the effective frequency range of the excitation (i.e., the range of frequencies producing improvement of the airfoil characteristics) depend strongly on the excitation amplitude.

Zaman [10] has reported significant differences in the optimum excitation parameters obtained in the various experiments. The results presented show a disagreement among the experimental data, with optimum Strouhal numbers varying by as much as an order of magnitude in some cases. An increase of the external excitation amplitude was found to result in a shift of the optimum effect towards the lower Strouhal numbers. Moreover, these Strouhal numbers were much lower than those predicted by linear instability theory. Zaman [10] concluded that this is due to nonlinear and viscous effects, especially in the presence of the separation bubble. However, no analysis has yet been done to support such a conclusion.

In most of the previous experimental studies cited above, attention was concentrated on lift and drag measurements and boundary-layer behavior. Wake measurements, if any, were done at a single downstream location, involving only the streamwise velocity component, and were only briefly related to the improvement of airfoil performance. However, wake structure is also an important aspect of airfoil performance. Recent experimental results of Huang and Lin [12] and Huang and Lee [13] suggest that vortex-shedding development and characteristics are closely related to boundary-layer behavior and shear-layer instability. In fact, Yarusevych et al. [14] show that optimum values of the control parameters may be correlated with airfoil boundary-layer 


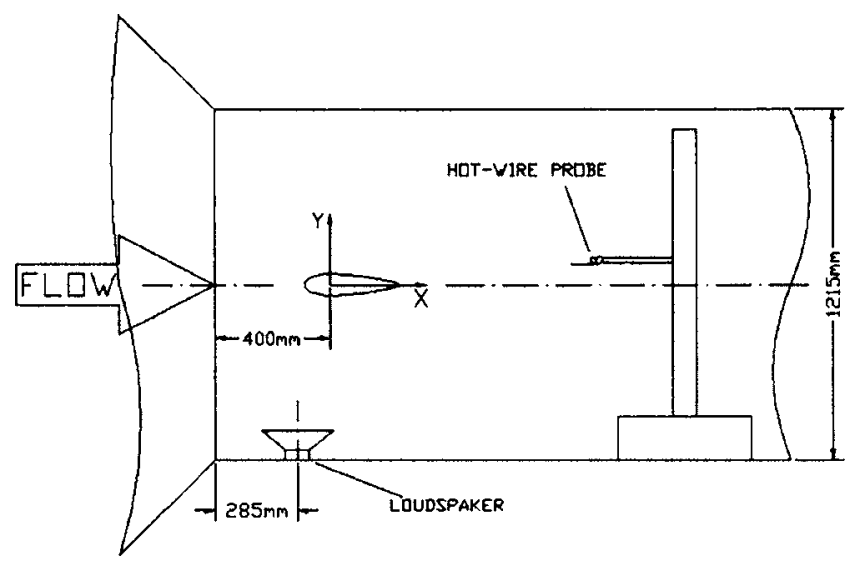

Fig. 1 Tunnel test section

and/or wake characteristics. It should be noted that the effect of airfoil vibrations has not been addressed in any of the cited references, even though such vibrations are likely to take place in most of the experiments involving acoustic excitation. In the light of this, the focus of the present work is to examine the effect of external acoustic excitation in the presence of structural vibrations on the boundary-layer separation and wake structure of a NACA 0025 symmetrical airfoil for various Reynolds numbers and angles of attack and to relate this effect to the improvement of airfoil performance.

\section{Experimental Setup}

Experiments were performed in a low-turbulence recirculating wind tunnel. The 5-m-long octagonal test section of this tunnel has a spanwise extent of $0.91 \mathrm{~m}$ and a height of $1.22 \mathrm{~m}$. The flow enters the test section through seven screens and a 9:1 contraction. The operating velocity $U_{0}$ is adjustable from 2.8 to $18 \mathrm{~m} / \mathrm{s}$, with a free-stream turbulence intensity level less than $0.1 \%$ within the bandwidth from 0.1 to $2500 \mathrm{~Hz}$. One wall of the test section is made of plexiglas for operational and visualization purposes. During the experiments, the free stream velocity was monitored by a pitot-static tube, with precision uncertainty estimated to be less than $2.5 \%$.

The performance of a symmetrical NACA 0025 aluminum airfoil with a chord length $c$ of $0.3 \mathrm{~m}$ and a span of $0.88 \mathrm{~m}$ was examined for a range of chord Reynolds numbers $\left(\operatorname{Re}_{c}\right)$ and three angles of attack $(\alpha)$. A schematic diagram of the test sections is shown in Fig. 1. The airfoil was mounted horizontally in the test section, $0.4 \mathrm{~m}$ downstream of the contraction. A Cartesian coordinate system was used, with $x$ and $y$ defined as shown in Fig. 1 and the origin at the centre of rotation of the airfoil.

Sound excitation was provided by means of a $250 \mathrm{~W}$ loudspeaker mounted on the test section floor, under the leading edge of the airfoil. The presence of the loudspeaker did not produce any measurable effect on the airfoil wake within the region of interest. The loudspeaker was driven through an amplifier by a variablefrequency wave generator and a foam-rubber pad could be installed to isolate the speaker from the test section floor. Thus, the mounting could be adjusted so as to either introduce vibrations to the structure along with sound waves or minimize the mechanical vibrations of the structure produced by the loudspeaker. A microphone and an accelerometer were employed to quantify sound excitation and airfoil surface vibrations, respectively. Based on the precision of the instruments for a frequency range of 40 to $1000 \mathrm{~Hz}$, the uncertainty of the microphone measurements is approximately $\pm 0.3 \mathrm{~dB}$, and that of the accelerometer data is less than $3 \%$.

Airfoil wake velocity data were obtained with constant temperature anemometers (Dantec 56C01 main units equipped with
56C17 CTA bridges). A Dantec 55P11 normal hot-wire probe and a Dantec 55P61 cross-wire probe were used separately to traverse vertical planes downstream of the airfoil. The probes were mounted on a remote-control traversing gear with a spatial resolution of $0.8 \mathrm{~mm}$. For accurate detection of vortex-shedding frequencies, the probe was positioned in the upper part of the wake at the $y / c$ locations corresponding to approximately one-half of the maximum velocity deficit for each Reynolds number and angle of attack. All hot-wire measurements were carried out in the vertical midspan plane of the tunnel. Based on the results of Kawall et al. [15], the maximum hot-wire measurement error was evaluated to be less than $5 \%$ and is attributed to the high turbulent intensities in the separated shear layer and near wake. Spectral analysis of the free-stream velocity signals with the model installed in the test section established that there was no periodicity associated with the approach flow.

For the spectral analysis of the velocity data sampled at $5000 \mathrm{~Hz}$, the duration of a sampled signal segment was chosen to be sufficiently large to provide a frequency resolution bandwidth of $0.61 \mathrm{~Hz}$, adequate for resolving narrow peaks in the spectrum. Based on the number of averages involved in obtaining the velocity spectra, the uncertainty of the spectral analysis was evaluated to be approximately $4.5 \%$.

To qualitatively visualize boundary-layer behavior, two rows of nylon tufts were installed on the airfoil surface $0.2 \mathrm{~m}$ away from the midspan. Each row contained ten $2-\mathrm{cm}-1$ ong tufts equally spaced from the leading to the trailing edge. The rows were separated by $2 \mathrm{~cm}$ in the spanwise direction and offset in the cordwise direction to increase spatial resolution.

\section{Experimental Results}

3.1 Flow Without Excitation. The results presented are for Reynolds numbers $\left(\operatorname{Re}_{c}\right)$ of $150 \times 10^{3}, 100 \times 10^{3}$, and $57 \times 10^{3}$ and angles of attack $(\alpha)$ of 10,5 , and 0 deg. All three values of $\operatorname{Re}_{c}$ are within the low Reynolds number region, i.e., $\operatorname{Re}_{c}<200$ $\times 10^{3}[1,2]$.

The surface flow visualization, which provided a qualitative picture of the boundary-layer behavior, showed that boundarylayer separation took place over a substantial portion of the upper surface of the airfoil at all three angles of attack for $\operatorname{Re}_{c}=57$ $\times 10^{3}$ and $\operatorname{Re}_{c}=100 \times 10^{3}$, with no boundary-layer reattachment. The separation occurred over approximately the aft $40 \%$ of the chord at $\alpha=0 \mathrm{deg}$, increasing to $50 \%$ at $\alpha=5 \mathrm{deg}$, and reaching $60 \%$ at $\alpha=10 \mathrm{deg}$ for these two Reynolds numbers. However, for $\operatorname{Re}_{c}=150 \times 10^{3}$, no clear evidence of boundary layer separation was found at $\alpha=0 \mathrm{deg}$ and $\alpha=5 \mathrm{deg}$, and only a small separation region was detected at the trailing edge, over the aft $10 \%$ of the chord, at $\alpha=10 \mathrm{deg}$. It should be noted that detailed boundarylayer measurements reported in [16] suggest that for this Reynolds number, the boundary layer separates on the upper surface of the airfoil and reattaches downstream to form a separation bubble over approximately $25 \%$ of the cord at all three angles of attack examined.

As a result of extensive boundary-layer separation for the two lower Reynolds numbers, wide wakes are formed behind the airfoil. Figure 2 depicts typical mean wake velocity profiles, i.e., plots of $\left(U_{0}-\bar{U}\right) / U_{0}$ versus $y / c$, for all the three Reynolds numbers investigated at $\alpha=10 \mathrm{deg}$. It is evident that the mean profiles for $\operatorname{Re}_{c}=57 \times 10^{3}$ and $\operatorname{Re}_{c}=100 \times 10^{3}$ differ significantly from the mean profile for $\operatorname{Re}_{c}=150 \times 10^{3}$. Specifically, the maximum velocity deficit of $0.24 U_{0}$ for $\operatorname{Re}_{c}=57 \times 10^{3}$ decreases to $0.21 U_{0}$ as the Reynolds number increases to $\operatorname{Re}_{c}=100 \times 10^{3}$. As the Reynolds number reaches $150 \times 10^{3}$, the wake narrows significantly with the maximum velocity deficit decreasing to $0.17 U_{0}$. Moreover, for $\operatorname{Re}_{c}=150 \times 10^{3}$, the location of the minimum velocity in the wake follows the incline of the trailing edge, similar to wakes of symmetrical airfoils at high Reynolds numbers. In contrast, for the two lower Reynolds numbers examined, the vertical location 


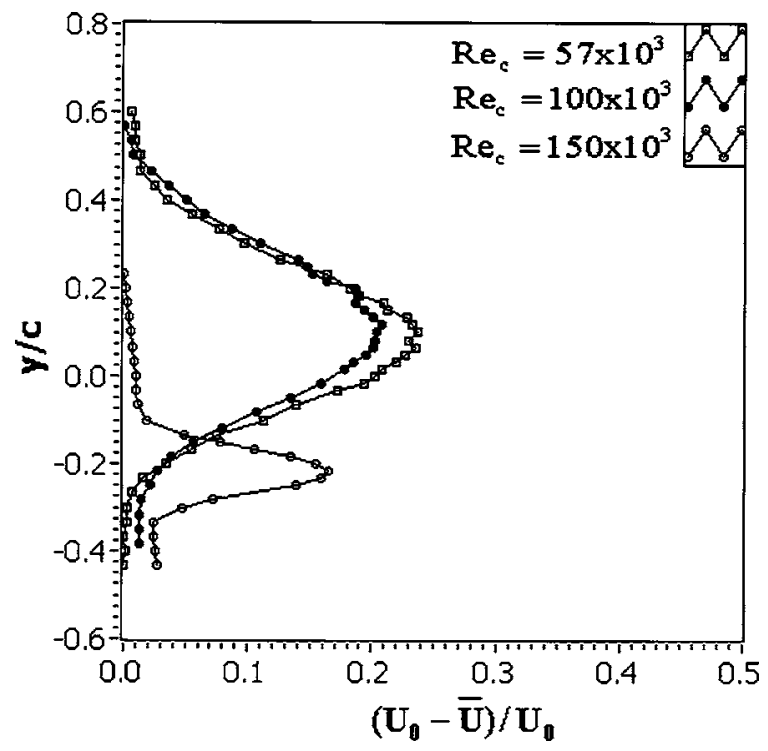

Fig. 2 Mean-velocity profiles, $x / c=1, \alpha=10^{\circ}$

of the minimum velocity in the wake is shifted upwards. This is attributable to boundary-layer separation on the upper surface of the airfoil, which governs wake formation and structure.

To gain insight into the airfoil wake structure, spectra of the wake velocity data are considered. Each of these spectra is normalized by the variance of the corresponding velocity component, so that the area under each spectrum is unity.

Figure 3 depicts spectra of the streamwise velocity component $\left(E_{u u}\right)$ at $x / c=2$ for all Reynolds numbers and angles of attack examined. Frequency is scaled with global parameters to form a Strouhal number, $\mathrm{St}_{d}=f d / U_{0}$, where $d$ is the vertical length of the airfoil projection on a cross-stream plane. For the case of $\operatorname{Re}_{c}$ $=57 \times 10^{3}$, a spectral peak centered at approximately $\mathrm{St}_{d}=0.21$ $(8 \mathrm{~Hz})$ at all three angles of attack is clear evidence of the existence of frequency-centered activity in the airfoil wake (Fig. 3(a)), attributable to vortex shedding. As the Strouhal numbers pertaining to the spectral peaks do not vary with angle of attack and compare well with the values reported for a circular cylinder $\left(\mathrm{St}_{d} \approx 0.2\right)$, vortex shedding for this Reynolds number appears to be similar to that of a bluff body.

The spectral results for $\operatorname{Re}_{c}=100 \times 10^{3}$ (Fig. 3(b)) reveal a degree of dependency of the vortex-shedding characteristics on angle of attack. At $\alpha=0 \mathrm{deg}$, a broad peak with a maximum at

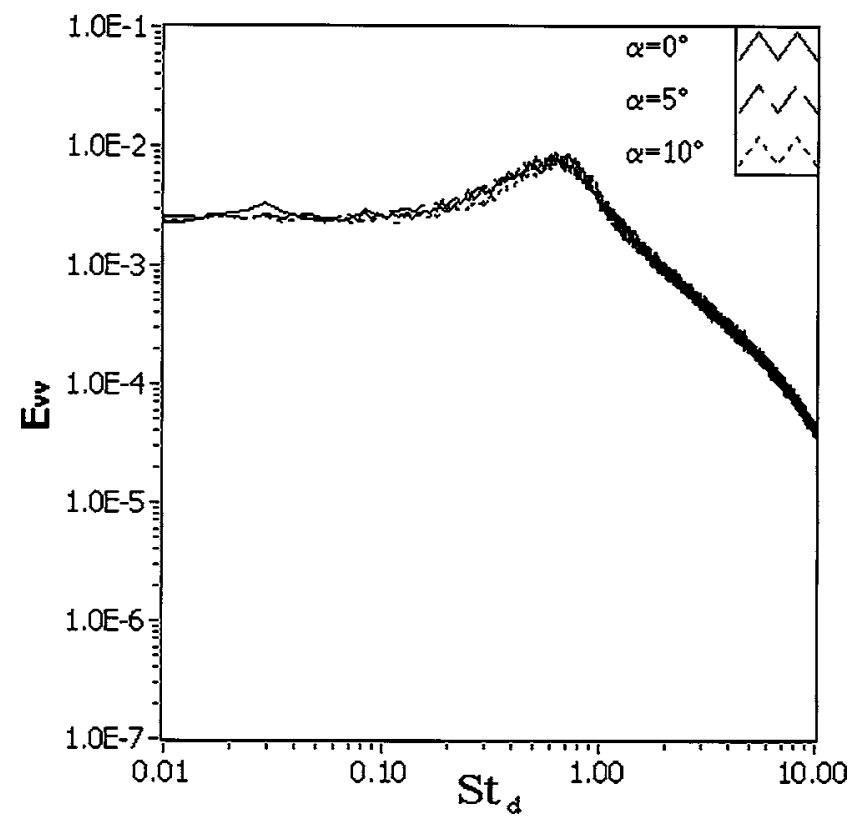

Fig. $4 E_{v v}$ spectra for $\mathrm{Re}_{c}=150 \times 10^{3}, x / c=3$

$\mathrm{St}_{d}=0.29(20 \mathrm{~Hz})$ is evident. As the angle of attack increases to $5 \mathrm{deg}$, a sharp peak centered at approximately $\mathrm{St}_{d}=0.29(20 \mathrm{~Hz})$ occurs. Finally, at $\alpha=10 \mathrm{deg}$, the vortex shedding appears to be similar to that in the wake of a circular cylinder, with the dominant spectral peak centered at $\mathrm{St}_{d}=0.22(15 \mathrm{~Hz})$. This indicates an increase in the length scale of the vortices, estimated as the ratio of free-stream velocity and the vortex-shedding frequency $\left(U_{0} / f\right)$.

A detailed wake survey carried out by means of a normal hotwire sensor did not reveal any evidence of frequency-centered activity in the airfoil wake for $\operatorname{Re}_{c}=150 \times 10^{3}$ at any angle of attack, with no distinct peaks revealed in the corresponding $E_{u u}$ spectra (Fig. 3(c)). This, however, does not rule out the existence of coherent structures; i.e., some sort of organized turbulent motion within the wake. As shown later for the two lower Reynolds numbers, the spectra of the vertical velocity component $\left(E_{v v}\right)$ are more sensitive to frequency-centered activity in wakes than spectra of the streamwise velocity component $\left(E_{u u}\right)$. Therefore, in an effort to determine whether some type of frequency-centered activity exists in the airfoil wake for $\operatorname{Re}_{c}=150 \times 10^{3}, E_{v v}$ spectra were measured at $x / c=3$. These spectra, presented in Fig. 4, display broad peaks, centered at approximately $\mathrm{St}_{d}=0.66(68 \mathrm{~Hz})$ at

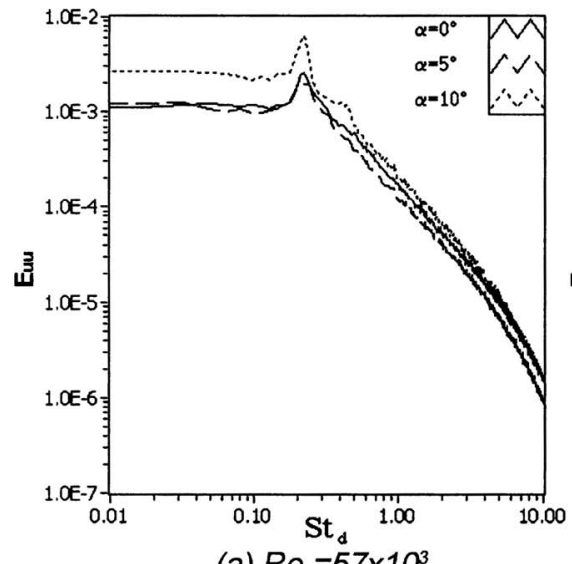

(a) $R e_{c}=57 \times 10^{3}$

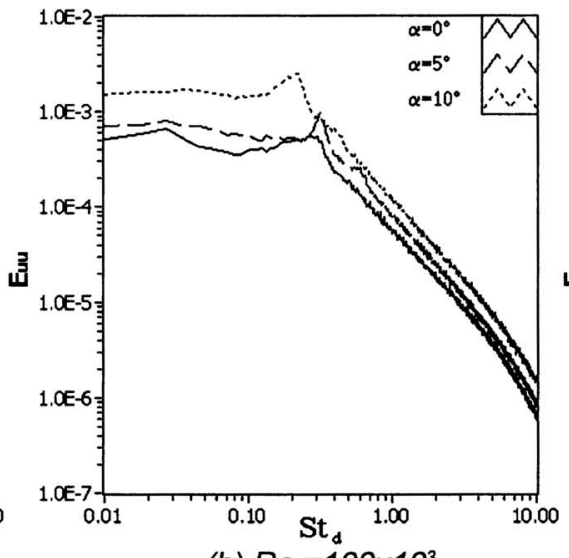

(b) $R e_{c}=100 \times 10^{3}$

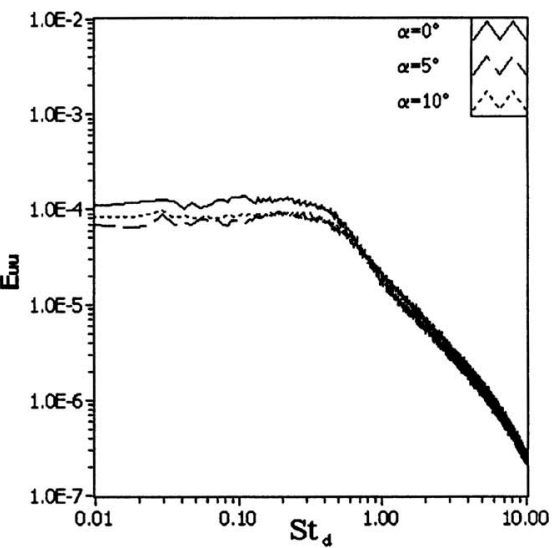

(c) $R e_{c}=150 \times 10^{3}$

Fig. $3 E_{u u}$ spectra, $x / c=2$ 


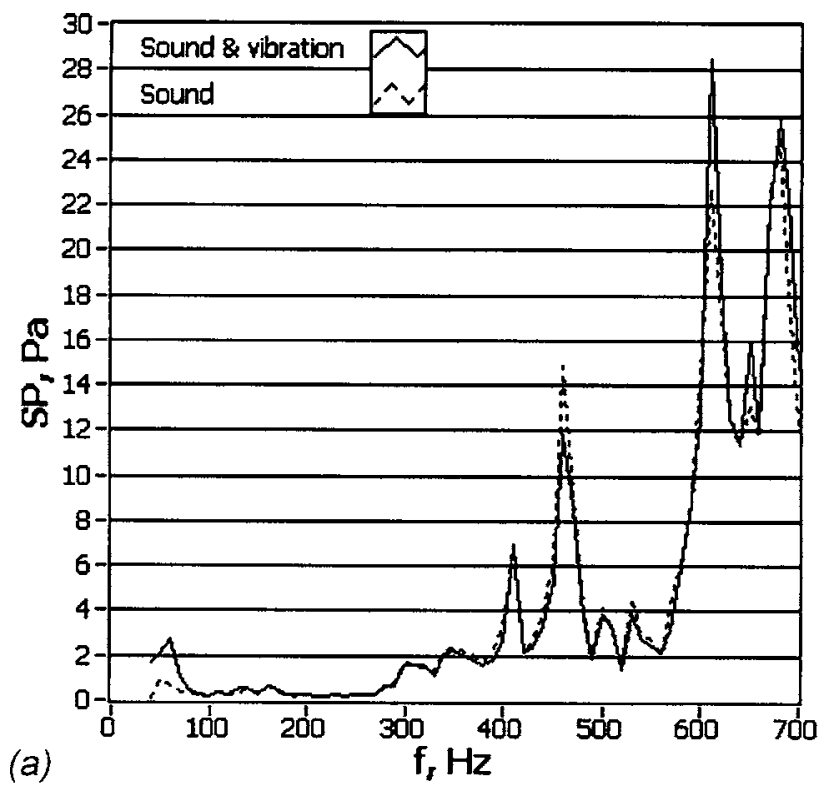

Fig. 5 Test section resonance characteristics: (a) sound pressure and (b) airfoil surface acceleration

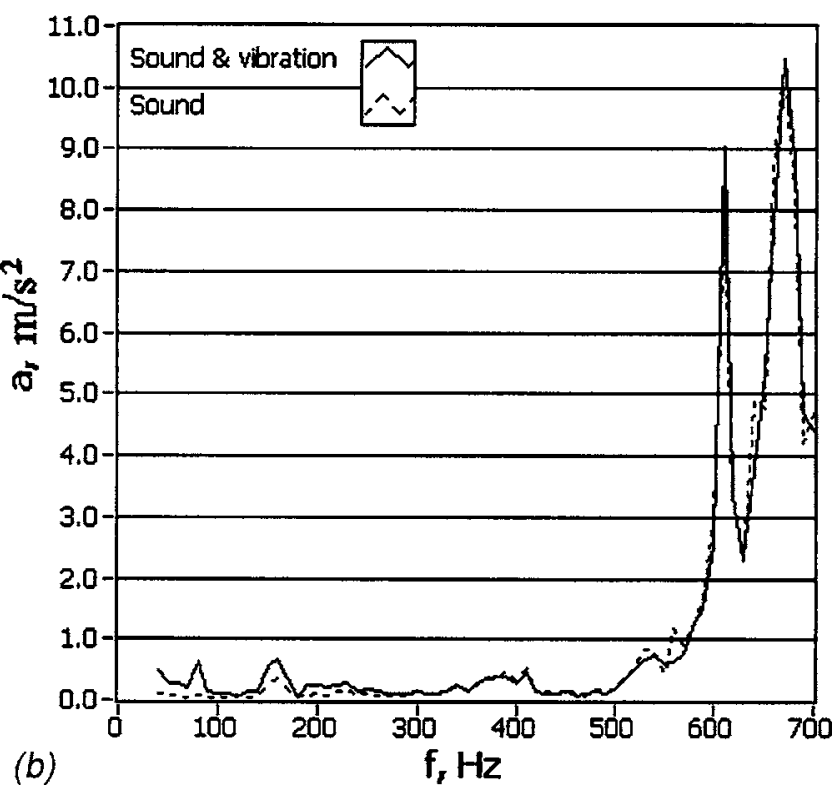

(b) all three angles of attack, revealing the presence of weak frequency-centered activity in the airfoil wake. It can be inferred that the coherence and length scale of the corresponding coherent structures are much lower than those of shed vortices detected for the two lower Reynolds numbers.

A distinct Reynolds number dependency of the wake structure characteristics can be detected from a comparison of Figs. 3 and 4. The vortex-shedding frequency steadily increases and length scale decreases as Reynolds number increases. Vortices become more diffuse and shedding less periodic, as peaks in the corresponding $E_{u и}$ spectra become broader and less defined with an increase of Reynolds number. Finally, the formation of the separation bubble on the airfoil surface for $\operatorname{Re}_{c}=150 \times 10^{3}$ results in the formation of much weaker coherent structures in the airfoil wake than the vortices observed for the two lower Reynolds numbers. Huang and Lin [12] and Huang and Lee [13] found a similar tendency in experiments with a NACA 0012 airfoil and reported the existence of a similar "transitional" regime of vortex shedding.

3.2 Excitation Parameters. Two types of excitation were considered: (i) "pure" acoustic excitation, with the loudspeaker isolated from the tunnel working section, and (ii) acoustic excitation with mechanical vibrations superimposed on the airfoil via the loudspeaker.

Since the effect of acoustic excitation depends on both excitation frequency and amplitude, wind tunnel resonance characteristics were examined at $U_{0}=0$. A microphone, positioned $40 \mathrm{~mm}$ above the upper surface at $x / c=0$ in the midspan of the airfoil, was used to measure sound pressure. Airfoil vibrations were monitored with an accelerometer mounted on the airfoil surface directly under the microphone. Sound pressure (SP) and vertical airfoil acceleration (a) were acquired with constant input voltage to the speaker and varying excitation frequency (Fig. 5). The results in Fig. 5(a) show substantial modulations in sound pressure at frequencies from approximately 280 to $700 \mathrm{~Hz}$ for both types of excitation examined. Distinct peaks located within this frequency range are associated with cross-resonances within the test section. A significant amplification of vibrations occurs at frequencies above $500 \mathrm{~Hz}$ (Fig. 5(b)), where acoustic resonance frequencies match natural frequencies of the model. Both sound pressure and acceleration variations are less substantial at frequencies below $280 \mathrm{~Hz}$.

Results in Fig. 5(b) suggest that the amplitude of airfoil surface vibrations for the case of acoustic excitation with structural vibrations is at least double that for the case of pure acoustic excitation in the range of 40 to $280 \mathrm{~Hz}$. However, the results show no substantial difference in acceleration for the two types of excitation at frequencies above $280 \mathrm{~Hz}$, where acoustic resonances take place. This is because vibrations at low frequency are less attenuated in the test section than those at higher frequencies. As a result, acoustic excitation with superimposed mechanical vibrations produces higher sound pressures at frequencies below about $100 \mathrm{~Hz}$ (Fig. 5(a)) and there is no substantial difference in sound pressures at higher frequencies.

To study the effect of acoustic excitation on airfoil performance, it is important to select an adequate parameter to quantify excitation amplitude. However, the choice of such a parameter is very problematic, as shown by Zaman et al. [5]. For example, their results question the suitability of sound pressure level or velocity fluctuations within the test section, as both are strongly affected not only by the resonances but also by the choice of the reference location, influence of the flow, and other factors. Following the approach taken in $[4,8,9]$, the effect of excitation frequency in the present study was investigated with a constant voltage supplied to the speaker, as it enables assessment of the effect of structural vibrations.

The effect of external acoustic excitation on the separated boundary layer was first examined by means of flow visualization. The flow was excited at different frequencies with constantamplitude voltage supplied to the speaker, in the presence of mechanical vibrations. For each of the cases examined, a range of frequencies (an effective-frequency range) was found to effect suppression of the separation region. As the amplitude of the excitation decreased, this range narrowed with boundary-layer reattachment finally occurring only at some "optimum" excitation frequency. Chang et al. [6] reported similar dependency of the effective-frequency range on the amplitude of the acoustic excitation. Moreover, a significant hysteresis in the suppression of the separation region by excitation was observed. Once reattachment occurred, the excitation frequency could be changed slightly from the optimum value or the excitation amplitude could be substantially lowered, with the boundary layer remaining fully attached.

The results summarized in Table 1 suggest that the optimum excitation frequency increases as Reynolds number or angle of attack increases. The effective-frequency range increases with an 
Table 1 Effective-frequency ranges and optimum frequencies of acoustic excitation in presence of mechanical vibrations

\begin{tabular}{|c|c|c|c|c|c|c|c|c|c|}
\hline \multirow[b]{2}{*}{$\operatorname{Re}_{c}$} & \multicolumn{3}{|c|}{$\alpha=0$ deg } & \multicolumn{3}{|c|}{$\alpha=5 \mathrm{deg}$} & \multicolumn{3}{|c|}{$\alpha=10 \mathrm{deg}$} \\
\hline & $\begin{array}{c}\text { Effect. } \\
\text { freq. } \\
\text { range } \\
(\mathrm{Hz})\end{array}$ & $\begin{array}{l}\text { Optimum } \\
\text { freq. }\end{array}$ & $\mathrm{St}_{d}$ & $\begin{array}{c}\text { Effect. } \\
\text { freq. } \\
\text { range } \\
(\mathrm{Hz})\end{array}$ & $\begin{array}{l}\text { Optimum } \\
\text { freq. }\end{array}$ & $\mathrm{St}_{d}$ & $\begin{array}{l}\text { Effect. } \\
\text { freq. } \\
\text { range } \\
(\mathrm{Hz})\end{array}$ & $\begin{array}{l}\text { Optimum } \\
\text { freq. }\end{array}$ & $\mathrm{St}_{d}$ \\
\hline $57 \times 10^{3}$ & $40-88$ & 65 & 1.71 & $70-80$ & 75 & 1.97 & $75-85$ & 80 & 2.07 \\
\hline $100 \times 10^{3}$ & $45-205$ & 117 & 1.70 & $75-220$ & 162 & 2.35 & $115-230$ & 169 & 2.42 \\
\hline $150 \times 10^{3}$ & $\ldots$ & $\ldots$ & $\ldots$ & $\ldots$ & $\ldots$ & $\ldots$ & $370-600$ & 450 & 4.27 \\
\hline
\end{tabular}

increase of Reynolds number, in agreement with [11], or a decrease of the angle of attack, similar to the trend reported in [7]. Note that all results in the table were obtained with a constant power input of $24 \mathrm{~W}$ to the speaker. Lack of correlation between the optimum excitation frequencies in Table 1 and acoustic resonance frequencies (Fig. 5(a)) suggest that excitation effect is not due to acoustic resonances in the test section. It should be noted, however, that the effective-frequency range results for $\mathrm{Re}_{c}=150$ $\times 10^{3}$ might be influenced by the acoustic resonances that occur between 400 and $500 \mathrm{~Hz}$.

The optimum excitation parameters obtained in the present study are in good agreement with those reported by Zaman and McKinzie [11] at low angles of attack $(\alpha \leqslant 6.5 \mathrm{deg})$. However, Strouhal numbers based on the optimum excitation frequency vary with Reynolds number and angle of attack (Table 1) and do not match corresponding values obtained for the vortex shedding frequency, being approximately an order of magnitude higher.

In order to gain insight into the control mechanism, spectra of the boundary layer velocity data were examined. Data were acquired in the unexcited flow at several $x / c$ locations within the transition region in the separated shear layer at $y / c$ positions that correspond to $0.5 \bar{U} / U_{0}$. Figure 6 depicts spectra of the streamwise velocity for $\operatorname{Re}_{c}=100 \times 10^{3}$ and $\operatorname{Re}_{c}=150 \times 10^{3}$ at $\alpha=10$ deg obtained downstream of the separation. For $\operatorname{Re}_{c}=100 \times 10^{3}$ (Fig. $6(a)$ ), a flat laminar flow spectrum is observed shortly past the separation point. As a separated shear layer develops downstream, a band of unstable Fourier components, sometimes referred to as a wave packet, occurs centered at $\mathrm{St}_{d}=2.44$, based on the fundamental frequency of the disturbances $f_{0}=170 \mathrm{~Hz}$. Further downstream, disturbances in this frequency band are substantially amplified and the band itself broadens, remaining centered at $\mathrm{St}_{d}$ $=2.44$; in addition, harmonics are generated. Rapid transition follows and a "classical" turbulent velocity spectrum is finally observed. A similar transition process is observed for $\operatorname{Re}_{c}=150$ $\times 10^{3}$ (Fig. 6(b)), with the band of naturally amplified disturbances observed at $\mathrm{St}_{d}=3.89\left(f_{0}=410 \mathrm{~Hz}\right)$.

To investigate a possible correlation between the acoustic excitation frequencies presented in Table 1 and those of the naturally amplified disturbances in the separated shear layer, the margins of the corresponding effective-frequency ranges are shown by dashed lines in Fig. 6. For both Reynolds numbers, the effectivefrequency range contains the frequencies of naturally amplified disturbances in the separated shear layer. Similar results were obtained for all the cases examined, suggesting that excitations at frequencies within the band around the fundamental frequency are effective. Moreover, the Strouhal numbers based on the fundamental frequencies correlate well with the corresponding values of $\mathrm{St}_{d}$ based on the optimum excitation frequency (Table 1). For all the cases examined, the maximum deviation of the optimum frequency from the fundamental frequency did not exceed $10 \%$, with the exception of $\operatorname{Re}_{c}=10 \times 10^{4}$ at $\alpha=0$ deg, where they differ by $20 \%$. It should be noted that these discrepancies are likely due to
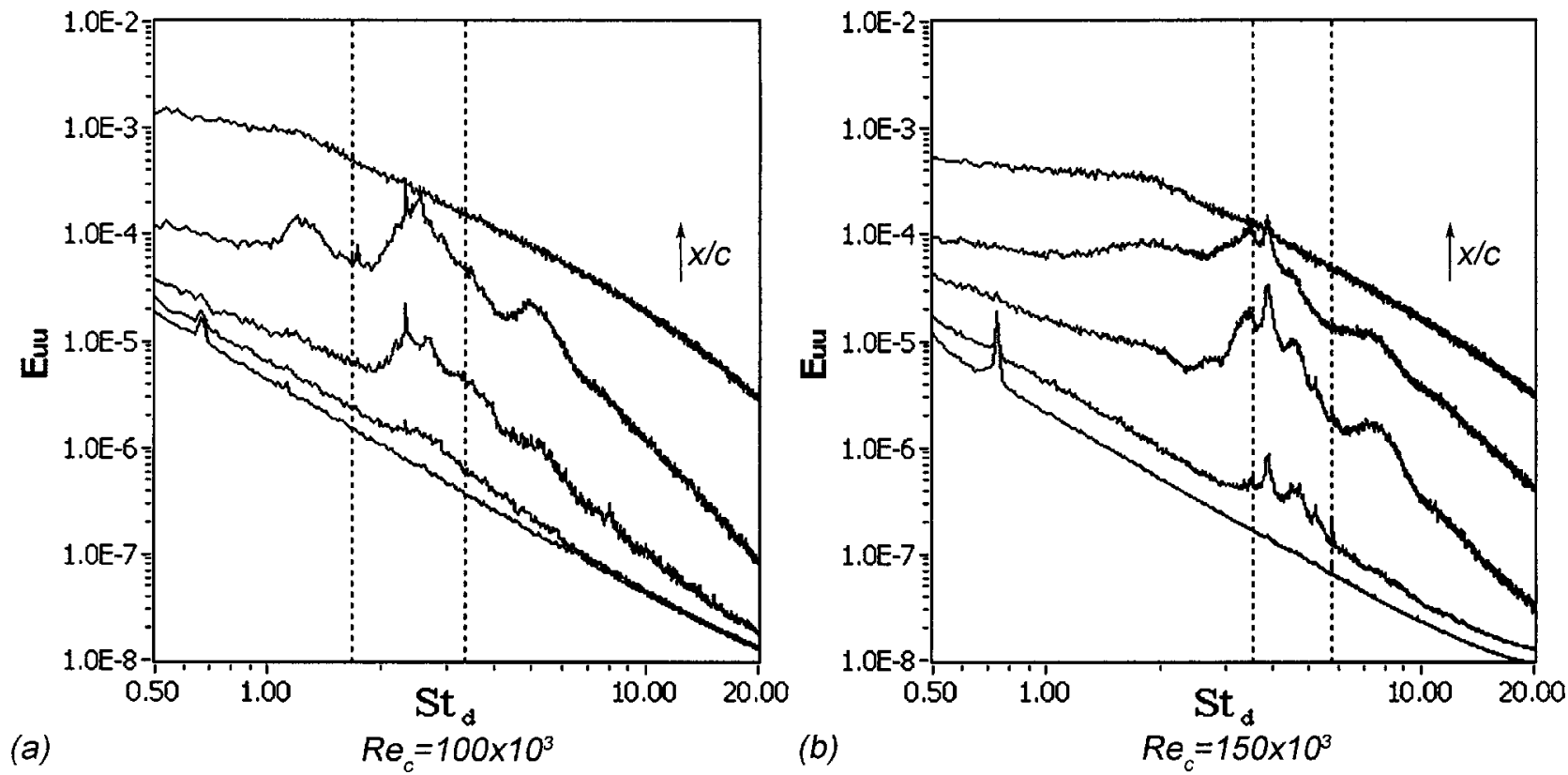

Fig. 6 Separated shear layer spectra, $\alpha=10$ deg; vertical dashed lines indicate the margins of the effective frequency ranges 


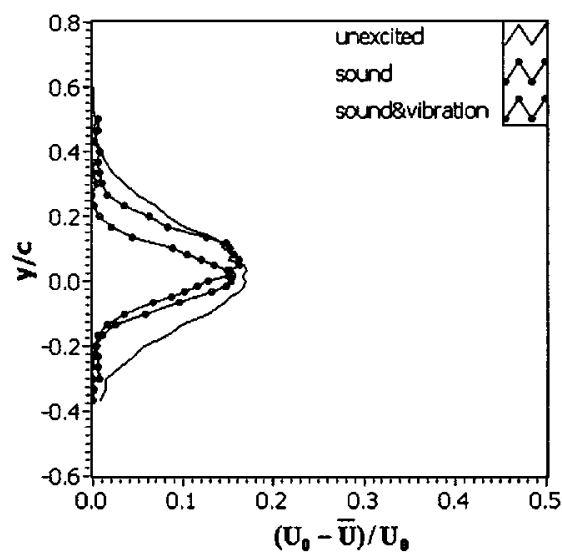

(a) $\alpha=0^{\circ}$

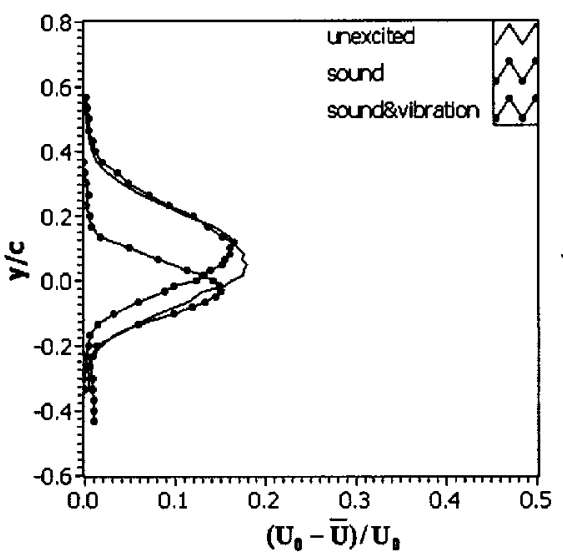

(b) $\alpha=5^{\circ}$

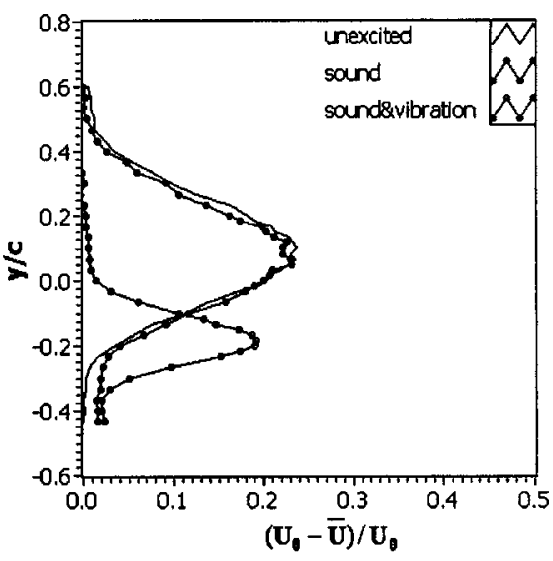

(c) $\alpha=10^{\circ}$

Fig. 7 Effect of excitation on mean profiles for $\mathrm{Re}_{c}=57 \times 10^{3}, x / c=2$

the combination of several factors, such as experimental uncertainties, rather than resulting from acoustic resonance alone. Therefore, it is concluded that matching the excitation frequency with the frequency of the most amplified disturbance in the separated shear layer is the optimum method of improving airfoil performance at a given Reynolds number and angle of attack. However, acoustic resonances should be taken into account, as they may influence the results if they are present within the frequency range of interest.

3.3 Excitation Effect. The effect of the two types of excitation on the airfoil performance and wake characteristics is considered below. Note that only results pertaining to excitation applied at the optimum frequencies (Table 1) and a constant power input of $24 \mathrm{~W}$ are considered henceforth.

Figure 7 shows $\left(U_{0}-\bar{U}\right) / U_{0}$ profiles with and without acoustic excitations for $\operatorname{Re}_{c}=57 \times 10^{3}$ at $x / c=2$. Acoustic excitation with superimposed mechanical vibrations significantly narrows the wake. Moreover, at $\alpha=5 \mathrm{deg}$ and $\alpha=10 \mathrm{deg}$, the wake is shifted down following the incline of the trailing edge. For example, at $\alpha=10 \mathrm{deg}$ (Fig. 7(c)), the maximum wake deficit is decreased by the excitation from $0.24 U_{0}$ to $0.2 U_{0}$ and its position is shifted from $y / c=0.1$ to $y / c=-0.2$. Pure acoustic excitation is much less effective in narrowing the wake for this Reynolds number. Comparison of the profiles in Fig. 5 suggests that this type of excitation affects mainly the lower part of the wake. The wake is more affected at $\alpha=0$ deg; i.e., it is diminished in size on both the upper and lower parts (Fig. 7(a)). At $\alpha=5 \mathrm{deg}$ (Fig. 7(b)), this effect is only seen on the lower half of the flow with the upper half unaffected; at $\alpha=10 \mathrm{deg}$ (Fig. 7(c)), the effect is slight. Comparison of the mean profiles for $\operatorname{Re}_{c}=100 \times 10^{3}$ at $x / c=2$ in Fig. 8 reveals significant narrowing of the wake at all three angles of attack for both types of excitation studied. In addition, the wake shifts down following the incline of the trailing edge at $\alpha=5 \mathrm{deg}$ and $\alpha$ $=10 \mathrm{deg}$ (Figs. $8(b)$ and $8(c)$ ), as in the case of the excitation with superimposed vibrations for $\operatorname{Re}_{c}=57 \times 10^{3}$. There is no significant difference in the effect from the two types of the excitation on the profiles in Fig. 8. Similar results were obtained for $\operatorname{Re}_{c}=150$ $\times 10^{3}$ at $\alpha=10 \mathrm{deg}$ and $x / c=2$. However, the extent of the effect was significantly smaller for this Reynolds number due to the suppression of a smaller separation region.

A quantitative analysis of the effect of acoustic excitation on airfoil performance is based on the drag coefficient results presented in Table 2. Sound pressure levels (SPLs) and normalized surface accelerations $(a / g)$ measured at the reference locations are also presented in the table. To facilitate data analysis, SPL and $a / g$ values shown for the two lower Reynolds numbers are averages based on measurements at the corresponding optimum frequencies. The drag coefficients are obtained by integration of the mean-wake profiles, as described in [17], with an uncertainty estimated to be less than 5\%. Note that the presence of the minor decrease of the freestream velocity at the lower part of the tunnel, which is less than $3 \%$ of $U_{0}$, was accounted for in calculating the drag coefficients. Moreover, results presented in Table 2 represent the relative reduction of airfoil drag coefficients $\left(C_{d}\right)$ with respect

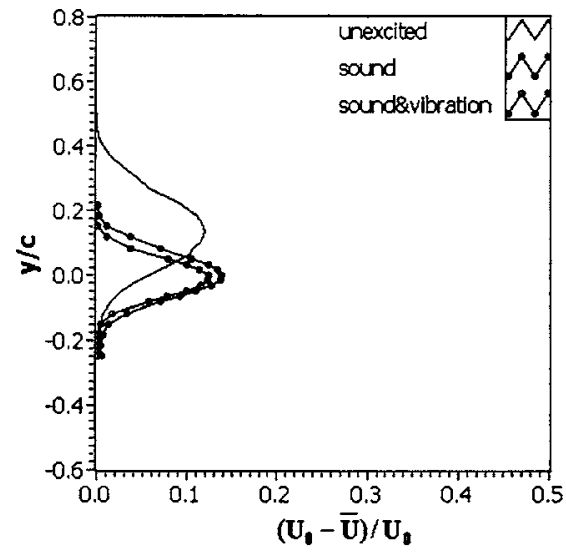

(a) $\alpha=0^{\circ}$

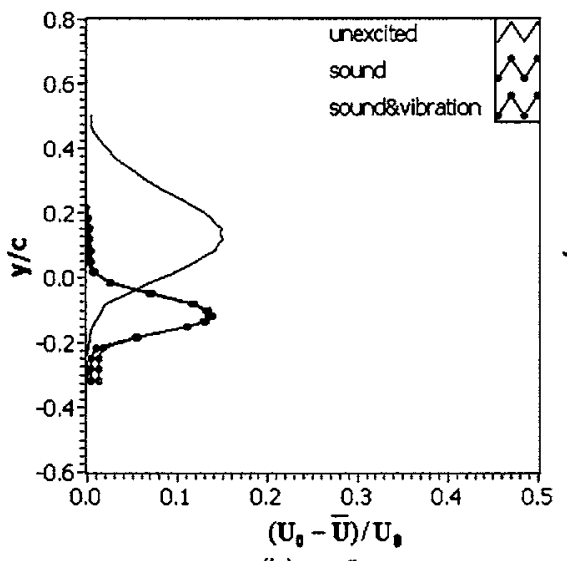

(b) $\alpha=5^{\circ}$

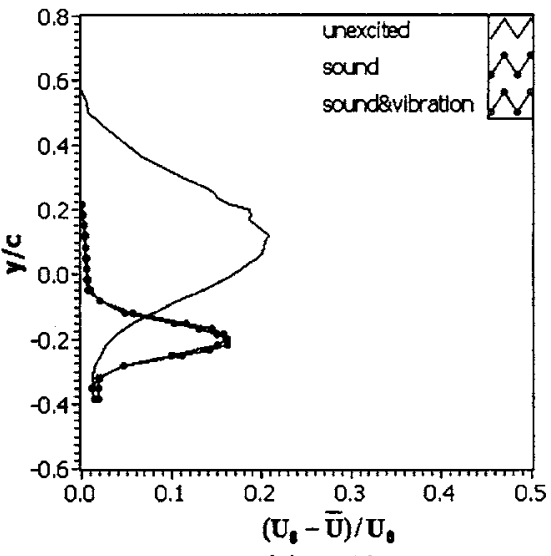

(c) $\alpha=10^{\circ}$

Fig. 8 Effect of excitation on mean profiles for $\operatorname{Re}_{c}=100 \times 10^{3}, x / c=2$ 


\begin{tabular}{|c|c|c|c|c|c|c|c|c|c|c|}
\hline \multirow[b]{2}{*}{$\operatorname{Re}_{c}$} & \multirow[b]{2}{*}{$\begin{array}{l}\mathrm{SPL}^{\mathrm{a}} \\
(\mathrm{dB})\end{array}$} & \multirow[b]{2}{*}{$\begin{array}{l}\mathrm{SPL}^{\mathrm{b}} \\
(\mathrm{dB})\end{array}$} & \multirow[b]{2}{*}{$a^{\mathrm{a}} / g$} & \multirow[b]{2}{*}{$a^{\mathrm{b}} / g$} & \multicolumn{2}{|c|}{$\alpha=0 \operatorname{deg}$} & \multicolumn{2}{|c|}{$\alpha=5 \mathrm{deg}$} & \multicolumn{2}{|c|}{$\alpha=10 \mathrm{deg}$} \\
\hline & & & & & $\frac{C_{d 0}-C_{d} \mathrm{a}}{C_{d 0}}$ & $\frac{C_{d 0}-C_{d} \mathrm{~b}}{C_{d 0}}$ & $\frac{C_{d 0}-C_{d} \mathrm{a}}{C_{d 0}}$ & $\frac{C_{d 0}-C_{d} \mathrm{~b}}{C_{d 0}}$ & $\frac{C_{d 0}-C_{d} \mathrm{a}}{C_{d 0}}$ & $\frac{C_{d 0}-C_{d} \mathrm{~b}}{C_{d 0}}$ \\
\hline $57 \times 10^{3}$ & 86 & 93 & 0.06 & 0.45 & 0.38 & 0.52 & 0.15 & 0.51 & 0.06 & 0.64 \\
\hline $100 \times 10^{3}$ & 86 & 87 & 0.19 & 0.35 & 0.30 & 0.44 & 0.61 & 0.61 & 0.75 & 0.75 \\
\hline $150 \times 10^{3}$ & 110 & 109 & 0.19 & 0.17 & $\cdots$ & $\cdots$ & $\cdots$ & $\cdots$ & 0.24 & 0.24 \\
\hline
\end{tabular}

${ }^{\mathrm{a}}$ Acoustic excitation only.

${ }^{b}$ Acoustic excitation with mechanical vibrations.

to the corresponding values of the drag coefficients calculated for the unexcited flow $\left(C_{d 0}\right)$, thus minimizing the effect of experimental uncertainty.

Clearly, the drag coefficients are lowered by either of the two types of excitation for all the cases examined. Note that acoustic excitation in the presence of structural vibrations produces higher sound pressure levels and more pronounced surface vibrations. There is a substantial difference between the effects of the two excitation methods for $\operatorname{Re}_{c}=57 \times 10^{3}$, with the acoustic excitation in the presence of structure vibrations being more effective. For instance, at $\alpha=10 \mathrm{deg}$, acoustic excitation of the flow with mechanical vibrations results in a decrease of the drag coefficient by $64 \%$, whereas pure acoustic excitation results in a decrease of only $6 \%$ (Table 2 ).

In contrast, for $\operatorname{Re}_{c}=100 \times 10^{3}$, the results suggest that there is little difference between the effects of two types of excitation on the airfoil performance. This correlates with insignificant dependency of the sound pressure level on the excitation type in this case. The most significant reduction of the drag coefficient is achieved for this Reynolds number, as the drag coefficient is reduced by $75 \%$ by both types of excitation at $\alpha=10 \mathrm{deg}$. Note that higher vertical accelerations that occur in the case of acoustic excitation with structural vibrations do not have a measurable effect on drag coefficient.

The results demonstrate that pure acoustic excitation is more efficacious for $\operatorname{Re}_{c}=100 \times 10^{3}$ than for $\operatorname{Re}_{c}=57 \times 10^{3}$, despite equivalent sound pressure levels in the test section for both Reynolds numbers. This implies that higher amplitude excitations are needed to influence the airfoil performance at lower Reynolds numbers. In addition for the two lower Reynolds numbers, the drag coefficient decrease, with the exception of the result for $\operatorname{Re}_{c}=57 \times 10^{3}$ at $\alpha=0 \mathrm{deg}$, becomes more pronounced as the angle of attack increases (Table 2). This trend is due to the sup- pression of the separation region, which increases as angle of attack increases. Ahuja and Burrin [9] reported a similar trend with respect to the effect of acoustic excitation on the lift coefficient.

The improvement of the airfoil performance for $\operatorname{Re}_{c}=150$ $\times 10^{3}$ is less significant than it is for the two lower Reynolds numbers. Nevertheless, a $24 \%$ decrease of the drag coefficient is achieved for both types of excitation. As in the case of $\operatorname{Re}_{c}$ $=100 \times 10^{3}$, both types of excitation produce equivalent sound pressure levels.

The results discussed above suggest that improvement of the airfoil performance is due to the amplification of the natural disturbances in the separated shear layer by periodic excitation applied at the fundamental frequency of these disturbances, which leads to boundary-layer transition and subsequent reattachment. Based on the comparative analysis of the velocity fluctuations introduced into the separated shear layer as a result of the airfoil surface vibrations and those due to acoustic excitation, it is concluded that acoustic waves have a dominant role in exciting the flow. However, structural vibrations need to be taken into account if acoustic excitation is utilized, as they can result in a higher sound pressure levels within the test section. This is especially important for flow control at lower Reynolds numbers, where higher excitation amplitudes and relatively low frequencies are required to influence airfoil performance.

To assess the effect of acoustic excitation on coherent structures in the airfoil wake, $v$-component velocity spectra are considered. It should be noted that peaks centered at the excitation frequencies and their harmonics appear in the $E_{v v}$ spectra for the excited flow.

Typical $E_{v v}$ spectra for $\operatorname{Re}_{c}=57 \times 10^{3}$ at $x / c=3$ are shown in Fig. 9. The sharp peak at $\mathrm{St}_{d}=0.21(8 \mathrm{~Hz})$ in the unexcited flow at 0 deg angle of attack (Fig. $9(a)$ ) is attenuated by the pure acoustic

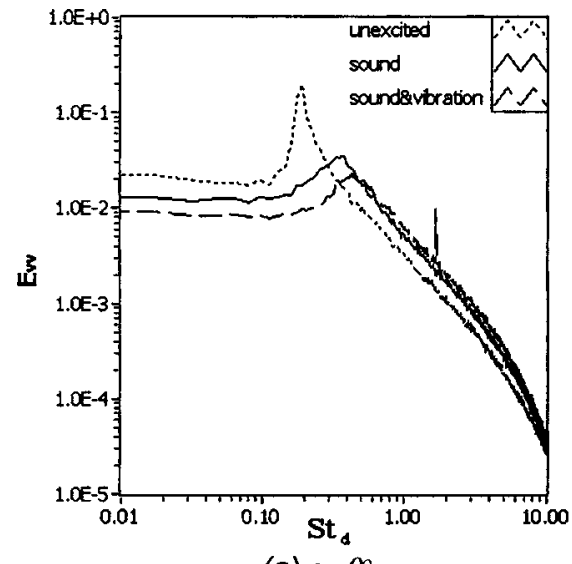

(a) $\alpha=0^{\circ}$

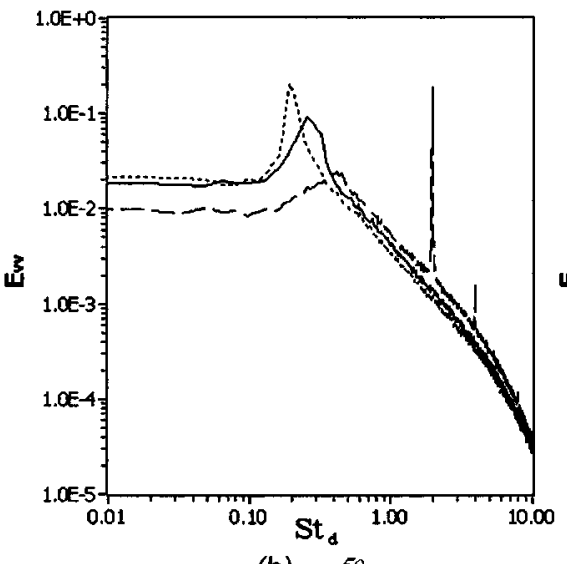

(b) $\alpha=5^{\circ}$

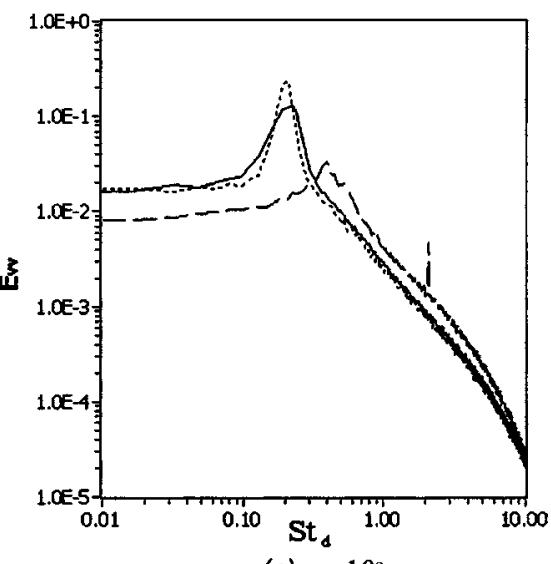

(c) $\alpha=10^{\circ}$

Fig. 9 Effect of excitation on $E_{v V}$ spectra for $\mathrm{Re}_{c}=57 \times 10^{3}, x / c=3$ 


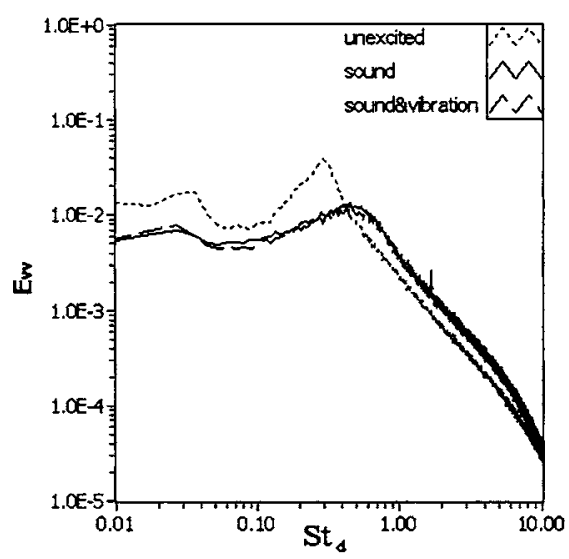

(a) $\alpha=0^{\circ}$

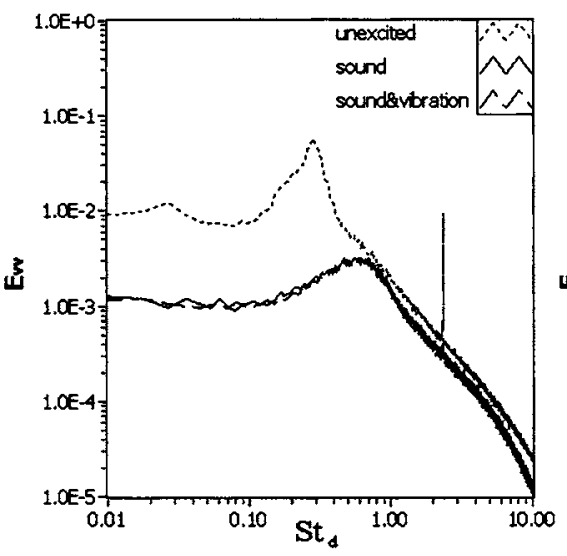

(b) $\alpha=5^{\circ}$

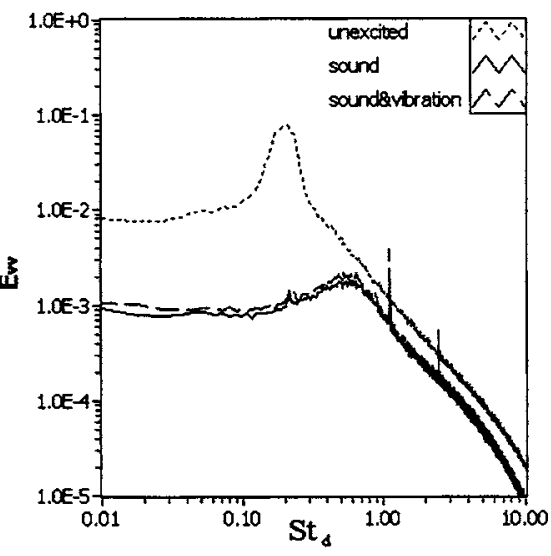

(c) $\alpha=10^{\circ}$

Fig. 10 Effect of excitation on $E_{v v}$ spectra for $\mathrm{Re}_{c}=100 \times 10^{3}, x / c=3$

excitation and is shifted to $\mathrm{St}_{d}=0.37(14 \mathrm{~Hz})$. An increase of the angle of attack to $5 \mathrm{deg}$ (Fig. $9(b)$ ) results in a slight attenuation of the peak, and it shifts to $\mathrm{St}_{d}=0.26(10 \mathrm{~Hz})$ from $\mathrm{St}_{d}=0.21$ $(8 \mathrm{~Hz})$. A further increase of the angle of attack to 10 deg results in the spectrum being only slightly altered by the acoustic excitation, with the peak shifted to $\mathrm{St}_{d}=0.22(8.5 \mathrm{~Hz})($ Fig. 9(c)). These observations suggest that excitation at optimum frequencies causes a decrease of the vortex length scale and coherence. The weakening of this effect with an increase of angle of attack is in good agreement with the results of Hsiao et al. [7]. As for the mean-wake velocity profiles, the effect of acoustic excitation with mechanical vibrations differs significantly from the effect of pure acoustic excitation for this Reynolds number (Fig. 9). The corresponding spectra, which are shown as long dashed curves, display diminished peaks centered at $\mathrm{St}_{d}=0.43(16.5 \mathrm{~Hz}), \mathrm{St}_{d}=0.41$ $(15.5 \mathrm{~Hz})$, and $\mathrm{St}_{d}=0.39(15 \mathrm{~Hz})$ at 0,5 , and $10 \mathrm{deg}$ angles of attack, respectively. The angle-of-attack effect is substantially reduced in this case, as the energy and the frequency of the peaks do not change significantly with an increase of $\alpha$.

Figure 10 shows $E_{v v}$ spectra for $\operatorname{Re}_{c}=100 \times 10^{3}$ at $x / c=3$. The peaks in the spectra associated with the unexcited flow are attenuated, broadened, and shifted to higher Strouhal numbers by either of the two types of the excitation studied. Note that the extent of these effects is much greater for this case than for the $\operatorname{Re}_{c}=57$ $\times 10^{3}$ case. The peaks corresponding to 0,5 , and 10 deg angles of attack that are centered at $\mathrm{St}_{d}=0.29(20 \mathrm{~Hz}), \mathrm{St}_{d}=0.29(20 \mathrm{~Hz})$, and $\mathrm{St}_{d}=0.22(15 \mathrm{~Hz})$ are strongly attenuated and shifted to $\mathrm{St}_{d}$ $=0.44(30 \mathrm{~Hz}), \mathrm{St}_{d}=0.58(40 \mathrm{~Hz})$, and $\mathrm{St}_{d}=0.57(40 \mathrm{~Hz})$, respectively. These peaks resemble those obtained in the unexcited flow for $\operatorname{Re}_{c}=150 \times 10^{3}$ (Fig. 4). Hence, due to the suppression of the separation region, the vortices shed in the airfoil wake when there is no excitation are replaced by coherent structures of substantially smaller length scale and coherence. As in the case of the mean velocity profiles, spectra for both types of excitation show significant overlap in Fig. 10 at corresponding angles of attack. This suggests that there is no substantial difference in the effect of these two types of excitation on the wake structure. The effect of the acoustic excitation on the vortex shedding for this Reynolds number is similar to the effect on the drag coefficient, as a more significant diminishment of the peaks in the spectra is achieved for higher angles of attack.

The results for $\operatorname{Re}_{c}=150 \times 10^{3}$ at $x / c=3$ and 10 deg angle of attack are presented in Fig. 11. As for $\operatorname{Re}_{c}=100 \times 10^{3}$, the broad peak centered at $\mathrm{St}_{d}=0.66(68 \mathrm{~Hz})$ in the spectrum pertaining to the unexcited flow is attenuated by both types of the excitation and is shifted to $\mathrm{St}_{d}=0.76(80 \mathrm{~Hz})$.

From the comparison of the spectral results and the drag coefficient results discussed previously, it is concluded that the mag- nitude of the acoustic excitation effect on the vortex shedding in the airfoil wake correlates with the improvement in the airfoil performance. Evidently, comparison of spectral results obtained with and without excitation can provide an assessment of the effect of acoustic excitation on airfoil performance.

\section{Conclusions}

Performance of a NACA 0025 airfoil at low Reynolds numbers was studied experimentally by means of hot-wire velocity measurements and complementary surface flow visualization.

Boundary-layer separation occurs on the upper surface of the NACA 0025 airfoil for all Reynolds numbers and angles of attack examined. For $\operatorname{Re}_{c}=150 \times 10^{3}$, the separated boundary layer reattaches to the airfoil surface, forming a laminar separation bubble. However, for $\operatorname{Re}_{c}=57 \times 10^{3}$ and $\operatorname{Re}_{c}=100 \times 10^{3}$, the separated shear layer fails to reattach to the airfoil surface, leading to the formation of much wider wakes compared to the case for $\operatorname{Re}_{c}$ $=150 \times 10^{3}$.

Coherent structures were detected in the airfoil wake for all Reynolds numbers and angles of attack examined. The character-

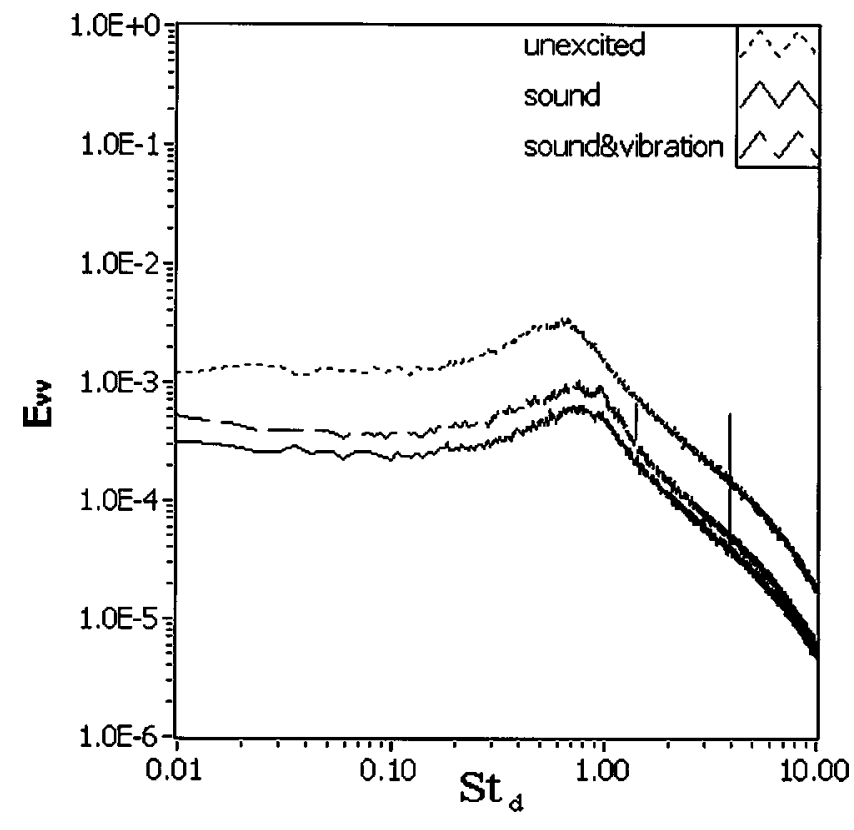

Fig. 11 Effect of excitation on $E_{v v}$ spectrum for $\operatorname{Re}_{c}=150$ $\times 10^{3}$ at $\alpha=10^{\circ}, x / c=3$ 
istics of these structures depend strongly on boundary-layer behavior and Reynolds number. Specifically, for the two lower Reynolds numbers examined, vortex shedding occurs in the airfoil wake at all angles of attack, with the associated Strouhal number comparable to that for vortex shedding in the wake of a circular cylinder. The vortex-shedding frequency increases and the characteristic length scale and coherency decrease as Reynolds number increases. Finally, the change in the boundary-layer behavior for $\operatorname{Re}_{c}=150 \times 10^{3}$, results in vortex shedding being replaced by the occurrence of much weaker coherent structures of substantially smaller length scale.

External acoustic excitation at particular frequencies and appropriate amplitudes with and without mechanical vibrations can substantially reduce or suppress the separation region so that a decrease in drag results. The effect of the excitation depends strongly on the excitation frequency and amplitude. In particular, the effective frequency range decreases with a decrease of the excitation amplitude. For a constant amplitude excitation, this range narrows with a decrease of the Reynolds number or increase of the angle of attack. The best effect is achieved at an optimum frequency, which increases as Reynolds number or angle of attack increases.

The results provide a definitive correlation between the optimum excitation frequencies and frequencies of naturally amplified disturbances in the separated shear layer. It is concluded that matching the excitation frequency with the frequency of the most amplified disturbance in the separated shear layer is the optimum method of improving airfoil performance at a given Reynolds number and angle of attack. The excitation promotes transition in the separated shear layer, effecting its reattachment, thus decreasing the size of the separation region.

It is concluded that acoustic waves play a dominant role in exciting the separated shear layer. However, low-frequency structural vibrations are found to have a significant effect on airfoil performance for $\operatorname{Re}_{c}=57 \times 10^{3}$, as they result in a higher sound pressure levels within the test section, and should be considered in future experiments involving acoustic excitations.

Acoustic excitation alters the vortex-shedding characteristics, decreasing the vortex length scale and the coherency of the vortex structure. Due to the suppression of the separation region, periodic vortex shedding in the airfoil wake is replaced by the occurrence of weak coherent structures. Moreover, the magnitude of the excitation effect on the wake structure correlates with the extent of the improvement in the airfoil performance, i.e., an increase in the lift and/or a decrease in the drag.

\section{Acknowledgment}

The authors gratefully acknowledge the Natural Sciences and Engineering Research Council of Canada (NSERC) for funding this work.

\section{Nomenclature}

$$
\begin{aligned}
a & =\text { airfoil surface acceleration } \\
C_{d} & =\text { airfoil drag coefficient }
\end{aligned}
$$

$$
\begin{aligned}
c= & \text { airfoil chord } \\
d= & \text { vertical length of airfoil projection on cross- } \\
& \text { stream plane } \\
E_{u u}= & \text { normalized energy spectrum of } u \\
E_{v v}= & \text { normalized energy spectrum of } v \\
f= & \text { frequency } \\
f_{0}= & \text { fundamental frequency of the disturbances } \\
g= & \text { acceleration due to gravity } \\
\operatorname{Re}_{c}= & \text { Reynolds number, } U_{0} c / v \\
\mathrm{SP}= & \text { sound pressure } \\
\mathrm{SPL}= & \text { sound pressure level } \\
\mathrm{St}_{d}= & \text { Strouhal number, } f d / U_{0} \\
U_{0}= & \text { free-stream velocity in the } x \text { direction } \\
\bar{U}= & \text { mean streamwise velocity } \\
u, v= & x \text { and } y \text { fluctuating velocity components } \\
x, y= & \text { streamwise and vertical coordinates } \\
\alpha= & \text { angle of attack } \\
v= & \text { kinematic viscosity of air }
\end{aligned}
$$

\section{References}

[1] Lissaman, P. B. S., 1983, "Low Reynolds Number Airfoils," Annu. Rev. Fluid Mech., 15, pp. 223-239.

[2] Mueller, T. J., and DeLaurier, J. D., 2003, “Aerodynamics of Small Vehicles," Annu. Rev. Fluid Mech., 35, pp. 89-111.

[3] Mueller, T. J., and Batill, S. M., 1982, "Experimental Studies of Separation on a Two-Dimensional Airfoil at Low Reynolds Numbers," AIAA J., 20(4), pp. 457-464.

[4] Nishioka, M., Asai, M., and Yoshida, S., 1990, "Control of Flow Separation by Acoustic Excitation,” AIAA J., 28(11), pp. 1909-1915.

[5] Zaman, K. B. M. Q., Bar-Sever, A., and Mangalam, S. M., 1987, "Effect of Acoustic Excitation on the Flow Over a Low-Re Airfoil," J. Fluid Mech., 182, pp. $127-148$.

[6] Chang, R. C., Hsiao, F. B., and Shyu, R. N., 1992, "Forcing Level Effect of Internal Acoustic Excitation on the Improvement of Airfoil Performance," J. Aircr., 29(5), pp. 823-829.

[7] Hsiao, F. B., Jih, J. J., and Shyu, R. N., 1997, "The Effect of Acoustics on Flow Passing a High-AOA Airfoil," J. Sound Vib., 199(2), pp. 177-178.

[8] Collins, F. G., and Zelenevitz, J., 1975, "Influence of Sound Upon Separated Flow Over Wings," AIAA J., 13(3), pp. 408-410.

[9] Ahuja, K. K., and Burrin, R. H., 1984, "Control of Flow Separation by Sound," AIAA Paper No. 84-2298.

[10] Zaman, K. B. M. Q., 1992, "Effect of Acoustic Excitation on Stalled Flows Over an Airfoil," AIAA J., 30(6), pp. 1492-1499.

[11] Zaman, K. B. M. Q., and McKinzie, D. J., 1991, "Control of Laminar Separation Over Airfoils by Acoustic Excitation,” AIAA J., 29(7), pp. 1075-1083.

[12] Huang, R. F., and Lin, C. L., 1995, "Vortex Shedding and Shear-Layer Instability of Wing at Low-Reynolds Numbers," AIAA J., 33(8), pp. 1398-1430.

[13] Huang, R. F., and Lee, H. W., 2000, "Turbulence Effect on Frequency Characteristics of Unsteady Motions in Wake of Wing," AIAA J., 38(1), pp. 87-94.

[14] Yarusevych, S., Kawall, J. G., and Sullivan, P. E., 2003, "Effect of Acoustic Excitation on Airfoil Performance at Low Reynolds Numbers," AIAA J., 41(8), pp. 1599-1601.

[15] Kawall, J. G., Shokr, M., and Keffer, J. F., 1983, “A Digital Technique for the Simultaneous Measurements of Streamwise and Lateral Velocities in Turbulent Flows," J. Fluid Mech., 133, pp. 83-112.

[16] Yarusevych, S., Sullivan, P. E., and Kawall, J. G., 2004, "Investigation of Airfoil Boundary Layer and Wake Development at Low Reynolds Numbers," AIAA Paper No. 2004-2551.

[17] Lu, B., and Bragg, M. B., 2000, "Experimental Investigation of Airfoil Drag Measurement with Simulated Leading-Edge Ice Using the Wake Survey Method," AIAA Paper No. 2000-3919. 